. IA-UR-96- 2091 CONF- $9606 / 10--5$

TITLE: AUTHOR(S):

SUBMITTED TO:
An electronic stopping power model for molecular dynamics and Monte Carlo simulation of ion implantation into silicon

$\mathrm{T}-11$

T-11

David Cai

JUL 191996 Niels Grønbech-Jensen Applied Theor. \& Condugtignal Physics T-11 University of Texas @ Austin University of Texas @ Austin
Charles M. Snell

Keith M. Beardmore

S. Morris

A.F. Tasch

$$
\text { RECENED }
$$




\section{DISCLAIMER}

This report was prepared as an account of work sponsored by an agency of the United States Government. Neither the United States Government nor any agency thereof, nor any of their employees, makes any warranty, express or implied, or assumes any legal liability or responsibility for the accuracy, completeness, or usefulness of any information, apparatus, product, or process disclosed, or represents that its use would not infringe privately owned rights. Reference herein to any specific commercial product, process, or service by trade name, trademark, manufacturer, or otherwise does not necessarily constitute or imply its endorsement, recommendation, or favoring by the United States Government or any agency thereof. The views and opinions of authors expressed herein do not necessarily state or reflect those of the United States Government or any agency thereof. 


\title{
An Electronic Stopping Power Model for Monte Carlo and Molecular Dynamics Simulation of Ion Implantation into Silicon
}

\author{
David Cai ${ }^{1}$, Niels Grønbech-Jensen ${ }^{1}$, Charles M. Snell ${ }^{2}$, Keith M. Beardmore ${ }^{1}$, Steven Morris ${ }^{3}$, and Al F. Tasch ${ }^{3}$ \\ 1 Theoretical Division, and ${ }^{2}$ Applied Theoretical and Computational Physics Division \\ Los Alamos National Laboratory, Los Alamos, New Mexico, 87545, U.S.A. \\ ${ }^{3}$ Microelectronics Research Center, The University of Texas at Austin, Austin, Texas, 78712, U.S.A.
}

\begin{abstract}
We develop a phenomenological model of electronic stopping power for modeling the physics of ion implantation into crystalline silicon. In the framework of effective charge theory, this electronic stopping power for an ion is factorized into (i) a globally averaged effective charge taking into account effects of close and distant collisions by target electrons with the ion, and (ii) a local charge density dependent electronic stopping power for a proton. This model is implemented into both molecular dynamics and Monte Carlo simulations. There is only one free parameter in the model, namely, the one electron radius $r_{s}^{\circ}$ for unbound electrons. By fine tuning this parameter, it is shown that the model can work successfully for both boron and arsenic implants. We report that the results of the dopant profile simulation for both species are in excellent agreement with the experimental profiles measured by secondary-ion mass spectroscopy (SIMS) over a wide range of energies and with different incident directions. This model also provides a good physically-based damping mechanism for molecular dynamics simulations in the electronic stopping power regime, as evidenced by the striking agreement of dopant profiles calculated in the molecular dynamics simulations with the SIMS data.
\end{abstract}

\section{INTRODUCTION}

Ion implantation in semiconductors is an important technology in integrated circuit device fabrication. A reliable description of as-implanted profiles and the resulting damage is needed for technological development. For semiconductor devices whose physical dimensions are of order of submicrons or smaller, low implant energies and reduction of thermal processing are necessary, resulting in more prominent channeling effects in the as-implanted profiles and less post-implant diffusion.

Study of the energy loss of channeled particles has a long history, for the channeling features can be used to elucidate the energy-loss mechanisms. Because of the channeling effect, electronic stopping power plays a much more significant role in ion implantation into crystalline solids than otherwise would be deduced from the application of the LSS theory to amorphous materials. It is especially true for heavy ion implants at low energies, such as arsenic ions in the energy range below $700 \mathrm{keV}$. Most of the existing Monte Carlo (MC) models are only concerned with boron implants, and have not modeled arsenic implants accurately with an electronic stopping power model consistent with that used for boron [1,2]. As will be shown below, the phenomenological model we developed for electronic stopping power can be implemented into a Monte Carlo simulation program for both boron and arsenic implants in different channels with equal success over a wide range of implant energies.

In addition to Monte Carlo simulations with the binary collision approximation (BCA), molecular dynamics (MD) incorporating multiple-interactions via many-body potentials can also be used to simulate the behavior of energetic ions in amorphous or crystalline silicon. This method is especially applicable at low energies, for which many-body, and multiple interactions are increasingly important. Therefore, MD results can be compared to BCA Monte Carlo simulations and used to check the validity of the low-energy BCA results. In order to achieve this, a correct description of the electronic stopping power should be incorporated into MD simulations, since, as shown in [3], traditional MD simulations produce extremely long channeling tails due to the absence of electronic stopping. An MD model has been developed incorporating the electronic stopping power as a damping mechanism [4]. This classical MD with the physically-based damping mechanism can generate dopant profiles in excellent agreement with experimentally-measured profiles obtained by secondary-ion mass spectroscopy (SIMS).

The phenomenological model we have developed for electronic stopping power is successfully implemented into both BCA Monte Carlo programs and MD simulations. Wide applicability requires that a model be valid for different implant species over a wide range of energies. We emphasize that this electronic stopping model is accurate both for boron and arsenic implants, thus providing a crucial test of the generality and validity of the model in capturing the correct physics of electronic stopping.

\section{THE MODEL}

According to the Brandt-Kitagawa (BK) theory [5], the electronic stopping power of an ion can be factorized into two components based on an effective charge scaling argument. One is the effective charge of the ion (if not fully ionized), $Z_{1}^{*}$, which is in general a function of ion velocity $v$ and the charge density of the target $\rho$, or equivalently, 
the one electron radius $r_{s}=[3 /(4 \pi \rho(\mathbf{x}))]^{1 / 3}$; the other is the electronic stopping power for a proton, $S_{p}\left(v, r_{s}\right)$. In the local density approximation, therefore, the total inelastic energy loss $\Delta E_{e}$ of an ion of constant velocity $v$ is $\Delta E_{e}=\int\left[Z_{1}^{*}\left(v, r_{s}\right)\right]^{2} S_{p}\left(v, r_{s}\right) d x$, where the integral is along the ion path. Since the effective charge is a continuous function of electronic density, mathematically, it is always possible to find a mean value, $r_{s}^{\circ}$ of $r_{s}$, such that $\Delta E_{e}$ can be rewritten as

$$
\Delta E_{e}=\left[Z_{1}^{*}\left(v, r_{s}^{0}\right)\right]^{2} \int S_{p}\left(v, r_{s}\right) d x .
$$

If the effective charge is a slowly varying function of space, physically, this means that $r_{s}^{0}$ describes an average number of unbound electrons in the sea and thus can be assumed to determine the Fermi surface. Therefore, we have the relation between the Fermi velocity and $r_{s}^{\circ}: v_{F}=1 /\left(\alpha r_{s}^{\circ}\right)$, where $\alpha=[4 /(9 \pi)]^{1 / 3}$ (Atomic units $e=\hbar=m_{e}=1$ are used throughout the paper unless otherwise specified). We note that this $r_{s}^{\circ}$ will be the only tunable parameter in our electronic stopping power model.

For an ion with $N=Z_{1}-Q$ bound electrons, where $Q$ is the charge number of the ion of atomic number $Z_{1}$, a variational approach minimizing the total energy of this partially ionized, moving projectile leads to the following dependence of the ion size $\Lambda$ on the ionization fraction $q=\left(Z_{1}-N\right) / Z_{1}[5]$

$$
\Lambda=\frac{2 a_{0}(1-q)^{2 / 3}}{Z_{1}^{1 / 3}[1-(1-q) / 7]},
$$

where $a_{0}=0.24005$. In the BK theory, the generalized Lindhard theory of the electronic stopping in a homogeneous electron gas with an electron density $n=3 /\left(4 \pi \bar{r}_{s}^{3}\right)$ is used. The total electronic stopping is estimated from the sum of the energy loss in soft, distant collisions, and the energy loss to the target electrons experiencing increased nuclear interaction in hard, close collisions. Then, an expression for the fractional effective charge of an ion is obtained [5],

$$
\gamma\left(\bar{r}_{s}\right)=q+C\left(\bar{r}_{s}\right)(1-q) \ln \left[1+\left(\frac{4 \Lambda}{\bar{r}_{s}}\right)^{2}\right],
$$

where $C\left(\bar{r}_{s}\right)$ is weakly dependent on the target and has a numerical value of about $1 / 2$. We will set $C=0.5$ below. Then, the effective charge is $Z_{1}^{*}=Z_{1} \gamma\left(\bar{r}_{s}\right)$. For our model, using the procedure (1) outlined above, this dependence of $\bar{r}_{s}$ is identified with the dependence of the mean value $r_{s}^{0}$. Therefore, the effective charge $Z_{1}^{*}$ has a nonlocal, i.e., spatially independent, character and depends on the Fermi surface. In light of the large amount of experimental data employed in [6] to extract an ionization scaling consistent with the BK theory, we will use this empirically verified scaling in our model, i.e.,

$$
q=1-\exp \left[-0.95\left(y_{r}-0.07\right)\right]
$$

where $y_{r}=v_{r} /\left(v_{B} Z_{1}^{2 / 3}\right)$, and

$$
\begin{array}{ll}
v_{r}=v_{1}\left(1+\frac{v_{F}^{2}}{5 v_{1}^{2}}\right) & \text { for } v_{1} \geq v_{F}, \\
v_{r}=\frac{3 v_{F}}{4}\left(1+\frac{2 v_{1}^{2}}{3 v_{F}^{2}}-\frac{v_{1}^{4}}{15 v_{F}^{4}}\right) & \text { for } v_{1}<v_{F} .
\end{array}
$$

In our model, the electronic stopping power for a proton is derived from a nonlinear density-functional formalism, which can be expressed as

$$
S_{p}\left(v, r_{s}\right)=-\left(\frac{d E}{d x}\right)_{\mathrm{R}} G\left(r_{s}\right)
$$

where

$$
\left(\frac{d E}{d x}\right)_{\mathbf{R}}=\frac{2 v}{3 \pi}\left[\ln \left(1+\frac{\pi}{\alpha r_{s}}\right)-\frac{1}{1+\alpha r_{s} / \pi}\right],
$$

and, for computational convenience, the correction factor $G\left(r_{s}\right)=1.00+0.717 r_{s}-0.125 r_{s}^{2}-0.0124 r_{s}^{3}+0.00212 r_{s}^{4}$ for $r_{s}<6$. We note that a different correction factor was used in Refs. [7,8], which does not have desired behavior for $r_{s} \ll 1$. Since the density functional result converges to the Ritchie formula (8) as $r_{s}$ decreases towards values sufficiently small compared to unity, this requires that the correction factor smoothly tend to unity as $r_{s} \rightarrow 0$. Obviously, the above $G\left(r_{s}\right)$ possesses the correct convergence property. The last ingredient needed for our model is the charge distribution $\rho(\mathbf{x})$ for silicon atoms in the crystal. We use the solid-state Hartree-Fock atomic charge distribution [6].

\section{BCA MONTE CARLO AND MD SIMULATION RESULTS}

First, in comparison with other electronic stopping models used in Monte Carlo simulations based on the MARLOWE platform, we stress that in our model the effective charge is a nonlocal quantity, neither explicitly dependent on the impact parameter nor on the charge distribution, and the stopping power for a proton depends on the local charge density of the solid. A purely nonlocal version of the BK theory was implemented into MARLOWE [7], in which both the effective charge and the stopping power for a proton depend on a single nonlocal parameter, namely, the averaged one electron radius. Its results demonstrated that energy loss for well-channeled ions in the $\mathrm{keV}$ region has high sensitivity to the oneelectron radius in the channel. It was pointed out that a correct density distribution is needed to account for the electronic stopping in the channel $[2,7]$. Later, a purely local version of the BK theory was developed to 
take into account the charge distribution of the electrons $[8,9]$. Good agreement between simulated dopant profiles and the SIMS profiles for boron implants into $\langle 100\rangle$ single crystal silicon was obtained. However, this purely local implementation of the BK theory did not successfully model the electronic stopping for the boron implants into the $\langle 110\rangle$ axial channel and arsenic implants as noted in [1].

In the present work, UT-MARLOWE was selected as the platform for our electronic stopping model implementation. In our simulations, we have turned off certain options, such as the damage model in the UT-MARLOWE code. In order to test the electronic stopping model we also used low dose $\left(10^{13} / \mathrm{cm}^{2}\right)$ implants for which the complication of damage effects on dopant profiles is not significantly involved. Also, for the simulation results we report below, $16 \AA$ native oxide surface layer, $300 \mathrm{~K}$ wafer temperature were used.

In the simulations, the free parameter $r_{s}^{\circ}$ was adjusted to yield the best results in overall comparison with the experimental data. The value $r_{s}^{\circ}=1.109 \AA$ was used for both boron and arsenic ions for all energies and incident directions. This value is physically reasonable for silicon. Note that the unbound electronic density in silicon with only valence electrons taken into account will give rise to a value of $1.061 \AA$ for $r_{s}$. The fact that our $r_{s}^{o}$ value is greater than $1.061 \AA$ indicates that not all valence electrons participate in stopping the ion as unbound electrons.

We display the Monte Carlo dopant profile simulation results as follows. We note in passing that the lower and upper limits of energy used in our simulations are determined by the energy range of the SIMS data available to us. In Figs. 1, 2 and 3, we show boron dopant profiles for the energies $15 \mathrm{keV}, 35 \mathrm{keV}$, and $80 \mathrm{keV}$ along $\langle 100\rangle$, $\langle 110\rangle$, and the off-axis direction with tilt $=7^{\circ}$ and rotation $=30^{\circ}$, respectively. It can be seen that the overall agreement with the SIMS data is excellent. In Fig. 1, the simulations show a good fit for the cutoff range. In the high energy regime, the simulated distribution shows a slightly peaked structure. This can be attributed to a strong channeling due to insufficient scatterings in the implants. We have noticed that by increasing, e.g., the native oxide layer thickness or divergence of the incident beam, the peak can be reduced. For the $\langle 110\rangle$ channeling case, the distribution indicates a possibility that the total electronic stopping power along the channel is a little too strong at the high energy end. However, it should be kept in mind that for this channel, the UT-MARLOWE model becomes sensitive to the multiple collision parameter which is employed as an approximate numerical correction for the effect of multiple overlapping nuclear encounters. It is not clear how to separate the contributions from these two different sources.

Figs. 4 and 5 show arsenic dopant profiles for energies ranging from $15 \mathrm{keV}$ to $180 \mathrm{keV}$ along $\langle 100\rangle$, and the offaxis direction with tilt $=8^{\circ}$ and rotation $=30^{\circ}$, respec-

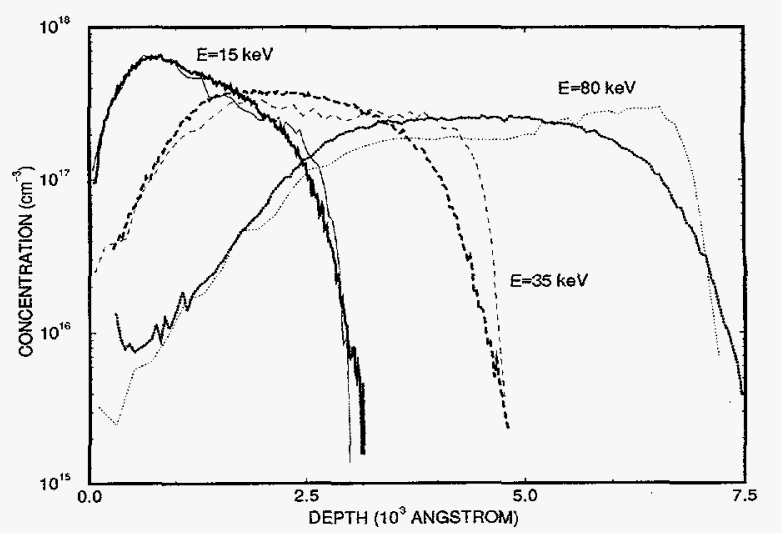

Fig. 1. Boron implant profiles for the $(100)$ direction with energies ranging from $15 \mathrm{keV}$ to $80 \mathrm{keV}$. Zero tilt and rotation angles. The thick lines are SIMS data.

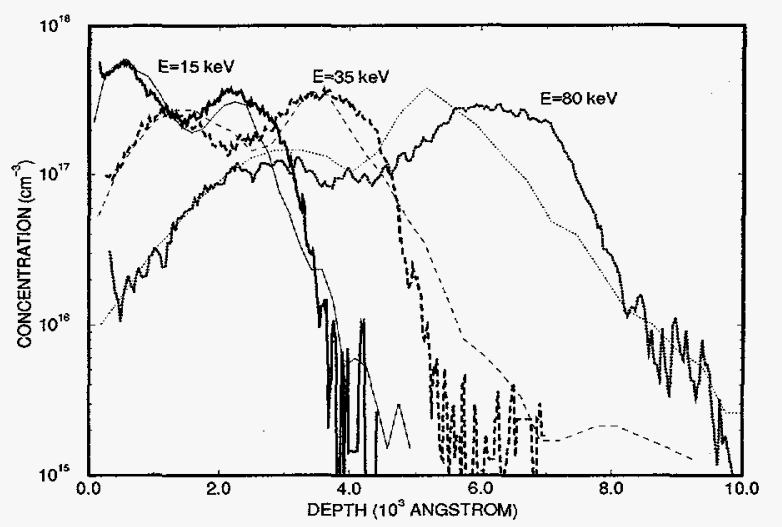

Fig. 2. Boron implant profiles for the $\langle 110\rangle$ direction with energies ranging from $15 \mathrm{keV}$ to $80 \mathrm{keV}$. Zero tilt and rotation angles. The thick lines are SIMS data.

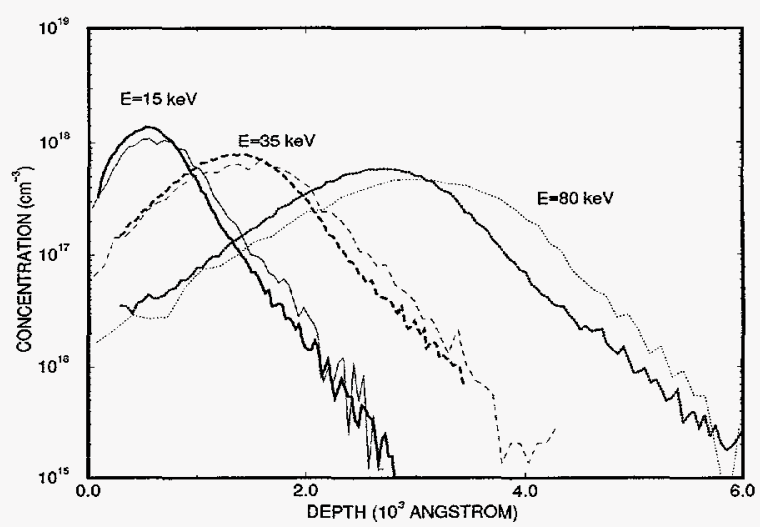

Fig. 3. Boron implant profiles for the $\langle 100\rangle$ direction with the tilt $=7^{\circ}$ and rotation $=30^{\circ}$. The thick lines are SIMS data.

tively. It can readily be concluded that our electronic model works successfully with arsenic as well as boron 


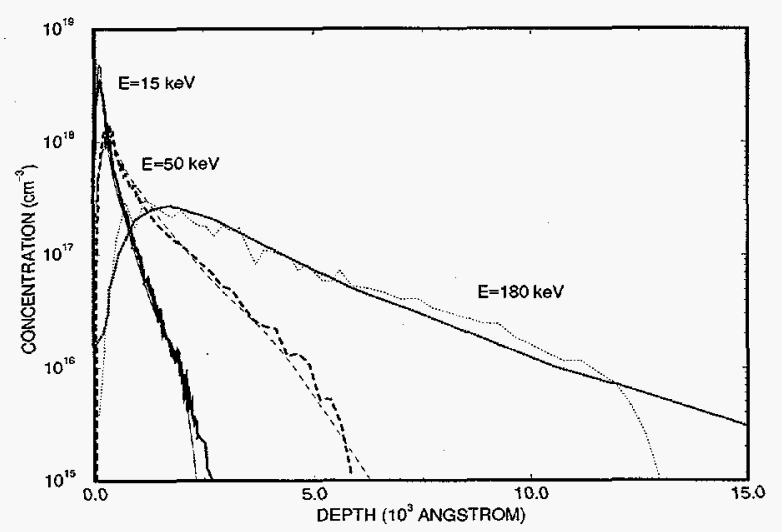

Fig. 4. Arsenic implant profiles for the $\langle 100\rangle$ direction with energies ranging from $15 \mathrm{keV}$ to $180 \mathrm{keV}$. Zero tilt and rotation angles. The thick lines are SIMS data.

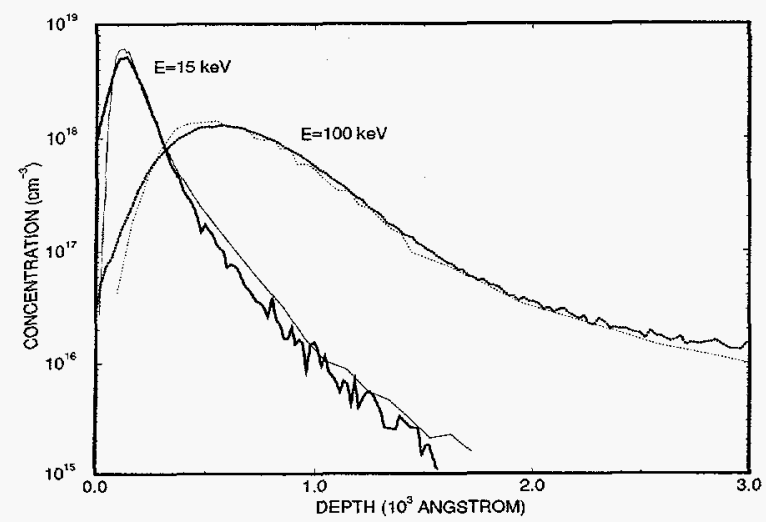

Fig. 5. Arsenic implant profiles for the $\langle 100\rangle$ direction with the tilt $=8^{\circ}$ and rotation $=30^{\circ}$. The thick lines are SIMS data.

implants into crystalline silicon.

Classical molecular dynamics simulation has been used to study the electronic stopping power as one of the damping mechanisms in the high energy regime. It has been demonstrated that experimental data, such as SIMS, can be used to test the validity of this physically-based damping model. The MD calculation results are in very good agreement with the experimental data, and with the $\mathrm{BCA}$ results [4]. This demonstrates that our electronic stopping power model provides a good physically-based damping mechanism for MD simulations of ion implantation.

\section{CONCLUSION}

We have developed a phenomenological electronic stopping power model for the physics of ion implantation. It has been implemented into MD and BCA Monte Carlo simulations. SIMS data have been used to verify this model in the MD and BCA Monte Carlo platforms. This model has only one free parameter, namely, the one electron radius of unbound electrons in the medium. We have fine tuned this parameter to obtain excellent results of dopant profiles compared with SIMS data in both MD and $\mathrm{BCA}$ Monte Carlo simulations. We emphasize that this model with a single parameter can equally successfully model both boron and arsenic implants into silicon over a wide range of energies and in different channeling and off-axis directions of incidence. This versatility indicates wide applicability of the model in simulations of other physical processes involving electronic stopping.

As discussed above, it is important to incorporate ionelectron couplings into MD simulations in the high energy radiation damage regime. It has been demonstrated that this model provides a crucial piece of physics in MD simulations for modeling energetic collisions in the electronic stopping power regime [4]. The agreement of the simulated dopant profiles with the SIMS data shows that the incorporation of this physically-based damping term into MD simulations is a phenomenologically reliable approach in the regime concerned. This agreement also suggests that MD can be used to generate dopant profiles for testing against the low energy $\mathrm{BCA}$ results when experimental data is not available.

\section{REFERENCES}

[1] S.H. Yang, S.J. Morris, S.Y Tian, K.B. Parab, and A.F. Tasch, "Monte Carlo simulation of arsenic ion implantation in (100) single-crystal silicon," IEEE Trans. Semiconductor Manufacturing, vol 9, no.1, pp. 49-58, 1996.

[2] C.S. Murthy and G.R. Srinivasan, "Computer simulation studies of ion implantation in crystalline silicon," IEEE Trans. Electron Devices, vol. 39, no. 2, pp. 264-273, 1992.

[3] N. Grønbech-Jensen, T. Germann, P.S. Lomdahl, and D.M. Beazley, "Molecular reconnaissance," IEEE Computational Science \& Engineering, vol. 2, no. 2, pp. 4-5, 1995.

[4] cf. contribution by K.M. Beardmore et al. in the proceedings of this conference.

[5] W. Brandt and M. Kitagawa, "Effective stopping-power charges of swift ions in condensed matter," Phys. Rev. B vol. 25, no. 9 pp. 5631-5637, 1982.

[6] J.F. Ziegler, J.P. Biersack and U. Littmark, The Stopping and Range of Ions in Solids, New York: Pergamon Press, 1985.

[7] N. Azziz, K.W. Brannon, and G.R. Srinivasan, "Electronic stopping power for low energy ions," in MRS Symp. Proc. 1985, vol. 45, pp. 71-76.

[8] K.M. Klein, C. Park, and A.F. Tasch, "Monte Carlo simulation of boron implantation into single-crystal silicon," IEEE Trans. Electron. Devices, Vol. 39, no. 7, pp. 16141621, 1992.

[9] K.M. Klein, C. Park, and A.F. Tasch, "Local electron concentration-dependent electronic stopping power model for Monte Carlo simulation of low-energy ion implantation in silicon," Appl. Phys. Lett. vol. 57, no. 25, pp. 2701-2703, 1990. 